\title{
Controlling the emission properties of multimode vertical-cavity surface-emitting lasers via polarization- and frequency-selective feedback
}

\author{
Y. Kouomou Chembo, ${ }^{1,2, *}$ Shyam K. Mandre, ${ }^{3}$ Ingo Fischer, ${ }^{4}$ Wolfgang Elsässer, ${ }^{3}$ and Pere Colet $^{2}$ \\ ${ }^{1}$ Institut FEMTO-ST (UMR CNRS 6174), Département d'Optique P. M. Duffieux, Université de Franche-Comté, \\ 16 Route du Gray, 25030 Besançon cedex, France \\ ${ }^{2}$ Instituto de Fisica Interdisciplinar y Sistemas Complejos IFISC (CSIC-UIB), Campus Universitat de les Illes Balears, \\ E-07122 Palma de Mallorca, Spain \\ ${ }^{3}$ Institute of Applied Physics, Darmstadt University of Technology, Schlossgartenstrasse 7, D-64289 Darmstadt, Germany \\ ${ }^{4}$ School of Engineering and Physical Sciences and Joint Research Institute of Integrated Systems, Heriot-Watt University, \\ Edinburgh EH14 4AS, United Kingdom
}

(Received 16 October 2008; published 21 January 2009)

\begin{abstract}
We investigate a polarization- and frequency-selective feedback scheme to control the emission properties of multimode vertical-cavity surface-emitting lasers. A joint experimental and theoretical approach is used to study the modal and polarization dynamics of the system, with an emphasis on the role of anisotropies. Both experiments and theory reveal that selective feedback of a single transverse mode results in a drastic increase of the selected mode's intensity. Furthermore, the influence of feeding back a single mode on the other transverse modes is discussed. Experimental measurements and numerical simulations also reveal that the emission and polarization dynamics can be considerably stabilized when this type of feedback is applied.
\end{abstract}

DOI: 10.1103/PhysRevA.79.013817

PACS number(s): 42.55.Px, 42.60.Jf, 06.30.Ft

\section{INTRODUCTION}

Since their first development in 1979 [1], vertical-cavity surface-emitting lasers (VCSELs) have gained considerable importance within the semiconductor laser market and are employed in various fields. The prominence of VCSELs can be attributed to advantageous emission properties and to the possibility to test them on chip. In particular, VCSELs exhibit single longitudinal-mode emission, almost circular output beams, and they can easily be integrated in twodimensional arrays. To achieve sufficient output power necessary for certain applications, VCSELs with aperture diameters of about $10 \mu \mathrm{m}$ or even larger have been developed. Such VCSELs exhibit multi-transverse-mode emission, which can deteriorate their spectral properties for applications. In addition, the multi-transverse-mode emission is associated with spatiotemporal dynamics on nanosecond and picosecond time scales [2]. Recent investigations have concentrated on the characterization of the emission dynamics of multimode VCSELs revealing the responsible mechanisms [2-7]. It is obvious that these emission dynamics give rise to performance deterioration or are even a hindrance to wider applications. The ultimate goal is therefore to control the emission properties of such VCSELs.

In order to experimentally control the emission properties of VCSELs, several schemes have been suggested and realized. These schemes include optical feedback configurations implementing spatial [8] or spectral filters [9], where single fundamental mode emission could be obtained up to certain pump currents. In addition, frequency-selective feedback has been implemented to realize cavity solitons and drifting excitations [10]. So far, the emphasis was put on controlling the VCSEL's spectral and spatial emission properties. However,

\footnotetext{
*Corresponding author; yanne.chembo@femto-st.fr
}

investigations of the influence of the feedback control on the spatiotemporal emission dynamics associated with multitransverse-mode emission are lacking. Moreover, in the case of frequency-filtered feedback, investigations on the effect of feeding back a single transverse mode on the other transverse modes have not been reported yet. Such extensive studies are essential, for both fundamental understanding of VCSEL emission and optimization of control techniques, especially considering the spatial effects prominent in multimode VCSELs' modal profiles.

In this paper we present joint theoretical and experimental investigations of the effect of frequency-filtered and spatially shaped feedback on the emission of a multi-transverse-mode VCSEL. With the feedback configuration applied here single transverse modes emitted by the VCSEL can be selectively enhanced. The influence of the enhancement of single modes on the emission properties are studied analytically, numerically, and experimentally. The investigations demonstrate that feedback of a single transverse mode leads to a rearrangement of the optical spectra depending on the polarization and the spatial profile of the fed back mode. The experimental investigations and theoretical analysis further show that the inherent intensity and polarization dynamics of the VCSEL can be considerably suppressed when feedback is applied.

The structure of the paper is as follows. In Sec. II we introduce the device and the experimental setup of the control scheme and furthermore, the applied techniques to characterize the emission behavior of the VCSEL. Section III contains the presentation of the obtained experimental results. After introducing the characteristics of the solitary VCSEL, we present the emission behavior of the VCSEL under the influence of the selective feedback scheme, focusing on the combined modal, polarization, and dynamical behavior. In Sec. IV we motivate and introduce the model that we use to describe the VCSEL under the influence of mode-selective 


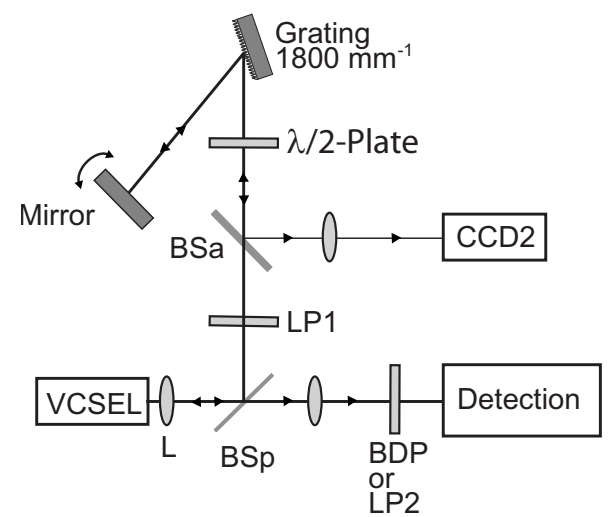

FIG. 1. Experimental setup to control the emission properties of a VCSEL employing frequency-filtered feedback. L, lens; LP, linear polarizer; BSp, beam splitter; BSa, beam sampler; and BDP, beam displacement prism. Detection includes power spectra of the intensity dynamics and single-shot measurements of polarization-(and spectrally) resolved near-field profiles.

feedback and the corresponding dynamical evolution equations. The analysis of these equations and the corresponding numerical modeling are presented in Sec. V. After a comparison of the results of experiment and modeling for the solitary laser, we present modeling results for the VCSEL with feedback. Finally, Sec. VI provides conclusions and an outlook of our paper.

\section{EXPERIMENTAL SETUP OF THE CONTROL SCHEME}

We employed an oxide-confined VCSEL emitting at a wavelength around $850 \mathrm{~nm}$ for our experimental studies. The oxide aperture exhibits a slight deformation such that the lengths of the aperture's principal axes amount to 9.8 and $8.8 \mu \mathrm{m}$, respectively. The VCSEL exhibits a threshold current of $\sim 1.3 \mathrm{~mA}$ and a maximum output power of about $2.5 \mathrm{~mW}$. Above threshold current, the output powers emitted by the two polarization orientations are similar. Therefore, we can directly observe the competition between the two polarization orientations emitted by this device.

In order to select a single transverse mode emitted by two-dimensional VCSEL structures, it is not sufficient to select and enhance the mode's spectral component. In addition, the fed back mode's beam profile needs to be matched to its emitted modal profile. The applied frequency-filtering feedback scheme must therefore enable selection of the spectral and spatial characteristics of the VCSEL's emission. Furthermore, due to the two orthogonal polarizations emitted by the VCSEL, it is desirable for the applied feedback scheme to allow for polarization-selective feedback.

Given the aforementioned requirements, we realized a frequency-, spatially, and polarization-filtering external resonator configuration, which is schematically depicted in Fig. 1. We used an aspheric lens (L) with a focal length of $f$ $=4.5 \mathrm{~mm}$ to collimate the VCSEL's emission beam, which was then directed towards a beam splitter (BSp). In the following, we will refer to the part of the beam being reflected (transmitted) by BSp as the feedback branch (detection branch). Using a linear polarizer (LP1) in the feedback branch, we select which of the two polarizations is fed back into the laser with an accuracy of about $2^{\circ}$. The beam is then spectrally dispersed and fed back by a Littman setup (a combination of a reflection grating with grating lines perpendicular to a projection plane and an external mirror) implemented in the feedback branch [11]. The efficiency of the grating (relation of the optical power in the first diffraction order to the incident power) is highest for the polarization orientation perpendicular to the grating lines. To ensure maximum efficiency $(\sim 70 \%)$ for either polarization selected by LP1, a $\lambda / 2$ plate can be inserted in the setup. We can therefore select either polarization orientation for feedback. On its propagation back towards the VCSEL, few percents of the spectrally dispersed beam in the feedback branch are reflected towards a charge-coupled device (CCD)-camera (CCD2) by a beam sampler (BSa). Using CCD2, the spectral component (transverse mode) being fed back into the laser can be monitored. When the $0^{\circ}\left(90^{\circ}\right)$ polarization is selected in the feedback branch, around $5 \%(8 \%)$ of the power contained in the selected mode is fed back into the VCSEL. The difference in feedback strength between the $0^{\circ}$ and $90^{\circ}$ polarization can be attributed to an asymmetry in the polarization-dependent reflectivity and transmittance of BSp. Due to the initial collimation of the beam by the aspheric lens L, the VCSEL's emission is imaged onto itself upon second propagation through $\mathrm{L}$ (autocollimation setup). Therefore, the beam profile of the fed back mode is matched to its emitted modal profile. The one-way optical path in the feedback branch (external optical cavity length) is about $57 \mathrm{~cm}$, implying a round-trip frequency of approximately $260 \mathrm{MHz}$

We use the beam in the detection branch to investigate the polarization dynamics and spatiotemporal emission dynamics of the VCSEL employing a fast-gated intensified CCD camera (4picos, Stanford Computer Optics, Inc.) with a temporal resolution (exposure time) of $2 \mathrm{~ns}$. We can acquire single-shot measurements of the VCSEL's near-field intensity profile by imaging the emission of the VCSEL facet onto the CCD chip. In addition, a beam displacement prism (BDP) can be used to spatially separate the two orthogonal polarizations, thus, allowing their simultaneous observation. Alternatively, we can spectrally disperse the near-field intensity profile emitted by the VCSEL using an imaging spectrometer [12]. By imaging the spectrally dispersed near fields onto the fast-gated camera we obtain single-shot measurements of polarization-resolved and spectrally dispersed nearfield profiles. Finally, we obtain polarization-resolved power spectra of the intensity dynamics using a linear polarizer (LP2) in combination with a fast avalanche photodiode (2.5 GHz $3 \mathrm{~dB}$ cutoff frequency), thus providing insight into the fast intensity dynamics. For the measurements presented in the following, the VCSEL was driven at a cw pump current of $4.5 \mathrm{~mA}$. The results could also be confirmed for lower pump currents.

\section{EXPERIMENTAL RESULTS}

\section{A. Nomenclature of the modes under study}

The transverse modes corresponding to the weakly guided optical cavity of the VCSEL are the so-called linearly polar- 
TABLE I. Nomenclature for the various characteristics of the transverse LP modes under study.

\begin{tabular}{|c|c|}
\hline \multicolumn{2}{|r|}{ Characteristics of transverse modes } \\
\hline \multicolumn{2}{|r|}{ (a) Order } \\
\hline Symbol & Denomination \\
\hline$m$ & $\begin{array}{l}\text { Azimuthal order: The mode has } 2 m \text { zeros } \\
\text { in the azimuthal direction }\end{array}$ \\
\hline$n$ & $\begin{array}{l}\text { Radial order: The mode has } n-1 \text { zeros } \\
\text { in the radial direction }\end{array}$ \\
\hline \multicolumn{2}{|r|}{ (b) Orientation } \\
\hline Symbol & Denomination \\
\hline$c$ & $\begin{array}{c}\text { Cosine mode: Maximum of intensity } \\
\text { along the } x \text { axis }(\varphi=0)\end{array}$ \\
\hline$s$ & $\begin{array}{l}\text { Sine mode: Minimum of intensity } \\
\text { along the } x \text { axis }(\varphi=0)\end{array}$ \\
\hline \multicolumn{2}{|r|}{ (c) Polarization } \\
\hline Symbol & Denomination \\
\hline$x$ & Horizontal $\left(\right.$ or $0^{\circ}$ ) linear polarization \\
\hline$y$ & Vertical (or $90^{\circ}$ ) linear polarization \\
\hline+ & Right circular polarization \\
\hline- & Left circular polarization \\
\hline
\end{tabular}

ized or $\mathrm{LP}_{m n}$ modes. Their spatial extension (typical diameter) approximately coincides with the cross section of the laser; they have $2 m$ zeros in the azimuthal direction, and $n$ -1 zeros in the radial one. Their orthogonal polarizations are denoted as $0^{\circ}\left(x\right.$ polarization) and $90^{\circ}$ ( $y$ polarization $)$, respectively. Therefore, in the whole paper, the subscripts $x$ or $y$ may be added to indicate the polarization state of the mode. Alternatively, right $(+)$ or left $(-)$ circular polarizations may be adopted instead of the $x$ and $y$ polarizations.

On the other hand, the $\mathrm{LP}_{m n}$ modes can also have different spatial orientations. In particular, their intensity may vary as $\cos ^{2} m \varphi$ or as $\sin ^{2} m \varphi$, where $\varphi$ is the azimuthal angle: in the first case, there are intensity maxima along the $x$ axis, while in the second case there are intensity minima along the same axis. We will therefore also label the $\mathrm{LP}_{m n}$ modes with the superscript $c$ (for cosine) or $s$ (for sine) to indicate their spatial orientation.

Hence, a LP mode is unequivocally characterized by its azimuthal $(m)$ and radial $(n)$ orders, its polarization $(x$ or $y$; + or - ), and its spatial orientation ( $c$ or $s$ ). These features are synthetically gathered in Table I and further details will be given in Sec. IV, which is, in part, devoted to the theoretical determination of these eigenmodes.

\section{B. Solitary VCSEL}

With the experimental setup described above, we have been able to characterize the modal and polarization dynamics of the solitary VCSEL when pumped well above threshold. For all measurements of the solitary VCSEL's emission, the feedback branch (cf. Fig. 1) was blocked.

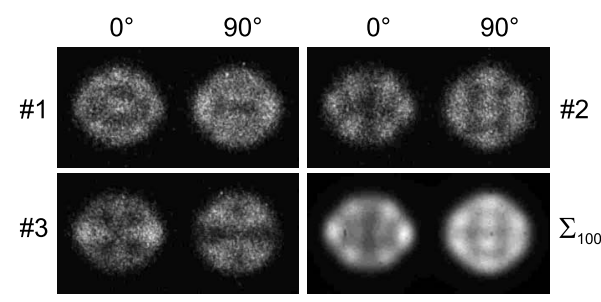

FIG. 2. Experimental single-shot measurements of the polarization resolved near fields at $I_{\text {pump }}=4.5 \mathrm{~mA}(\# 1 \cdots \# 3)$ and sum over 100 such single-shot measurements $\left(\Sigma_{100}\right)$. The laser is in the solitary emission configuration.

Figure 2 depicts four images of the solitary VCSEL's polarization-resolved near-field intensity profiles. The images \#1-\#3 depict single-shot measurements of the near-field profiles and $\Sigma_{100}$ depicts a calculated sum of 100 different single-shot measurements. The structured profiles visible in images \#1-\#3 indicate multiple high-order transverse-mode emission. Moreover, the three images exhibit varying spatial intensity distributions, thus revealing the presence of intensity and polarization dynamics in the VCSEL's emission. Spatial complementarity between the intensity distributions of the two polarizations can be observed, as local intensity maxima in one of the polarizations correspond to local intensity minima in the other polarization and vice versa. The polarization-resolved near-field profile in $\Sigma_{100}$ also exhibits the spatial complementarity between the two polarizations. We can therefore state that the transverse modes are not emitted randomly in the two polarizations. Instead, it seems that individual modes preferably emit in one of the two polarizations.

Next, we have performed polarization- and spectrally resolved near-field measurements. The corresponding singleshot profiles of the transverse modes are depicted in Fig. 3, which reveals the emission of a multitude of transverse modes in both polarizations. The vertical axis depicts a transverse (radial) dimension while the horizontal axis depicts the spectral dispersion of the near-field intensity distribution.
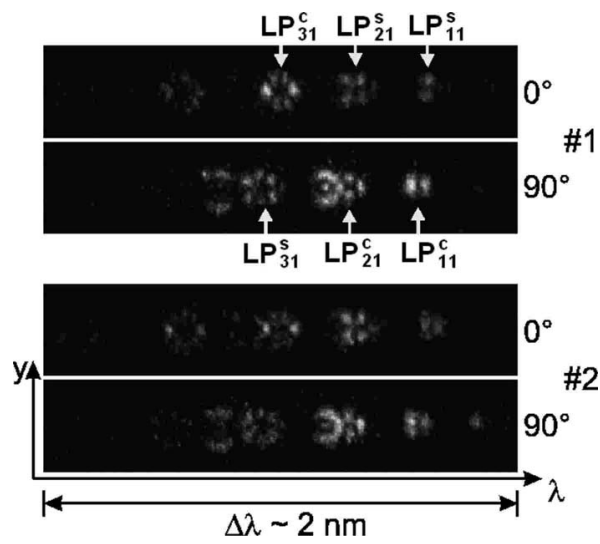

FIG. 3. Experimental single-shot measurements of spectrally resolved near-field intensity distributions. One transverse dimension is depicted in the vertical direction, while the horizontal direction exhibits the spectral dispersion maintaining the spatial resolution. The VCSEL was operated at a cw pump current of $4.5 \mathrm{~mA}$, in the solitary emission configuration. 


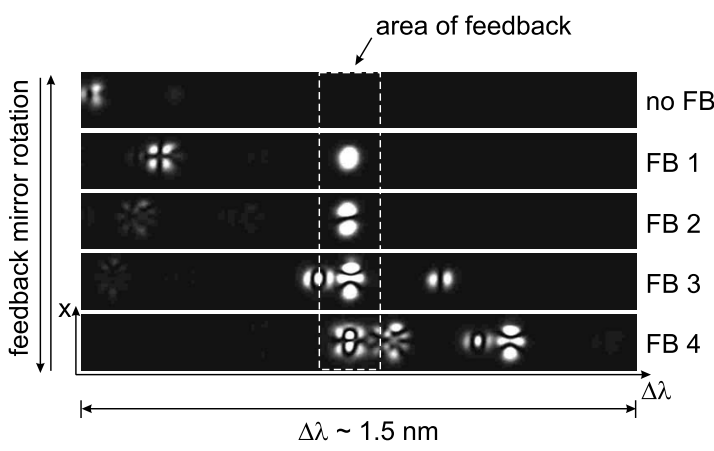

FIG. 4. Experimental monitoring of the fed back mode with CCD2 (cf. Fig. 1). The mode within the area illustrated by the dashed rectangle is selected. By rotation of the external mirror, different transverse modes can be selected. Note that the ordinate is $x$ in this plot.

Obtaining such optical spectra has the advantage that spatial resolution is maintained even in the horizontal direction. Spatially overlapping modes compete for the available spatial gain, which results in anticorrelation of these modes; in contrast, spatially complementary modes are rather correlated [7]. For example, such mode competition can be seen in the case of the $\mathrm{LP}_{11}$ modes in spectra \#1. Here, $\mathrm{LP}_{11}^{c}$ $\left(\mathrm{LP}_{11}^{s}\right)$ denotes the $\mathrm{LP}_{11}$ mode whose intensity maxima are aligned along the $x$ axis ( $y$ axis). Furthermore, there is a mode competition between the spatially complementary modes $\mathrm{LP}_{31}^{c}$ and $\mathrm{LP}_{31}^{s}$, which are active in the two polarizations (e.g., spectra \#1): a local intensity minimum in the $0^{\circ}$ polarization corresponds to a local intensity maximum in the $90^{\circ}$ polarization and vice versa.

For the single-shot measurements in Fig. 2 (images \#1\#3) and Fig. 3, the exposure time of the fast-gated CCD camera was chosen as 2 ns. The spatiotemporal intensity dynamics revealed by the polarization resolved near-field profiles therefore suggest that the polarization dynamics must have contributions on time scales slower than the 2 ns exposure time. This polarization dynamics has been studied in detail previously [2,7] and needs to be considered in polarization-sensitive applications. It is therefore of significant interest to control the polarization dynamics along with the spatial modes.

\section{VCSEL in a frequency-selective feedback scheme}

Frequency-filtered feedback is applied to the VCSEL by using the feedback branch introduced in Fig. 1. The dashed rectangle in Fig. 4 essentially represents the spatial and spectral area of feedback, as described in Sec. II. Rotation of the mirror in the Littman setup allows us to scan the spectrally dispersed emission across the VCSEL aperture and selectively feed back single transverse modes. They then appear in the area of feedback recorded via CCD2. Selection and feedback of a certain mode leads to significant enhancement of its intensity. To reveal the influence of the feedback on the emission dynamics of the VCSEL, we simultaneously acquired in Fig. 5 single-shot measurements of polarizationresolved and spectrally dispersed near-field profiles (as in Fig. 3).

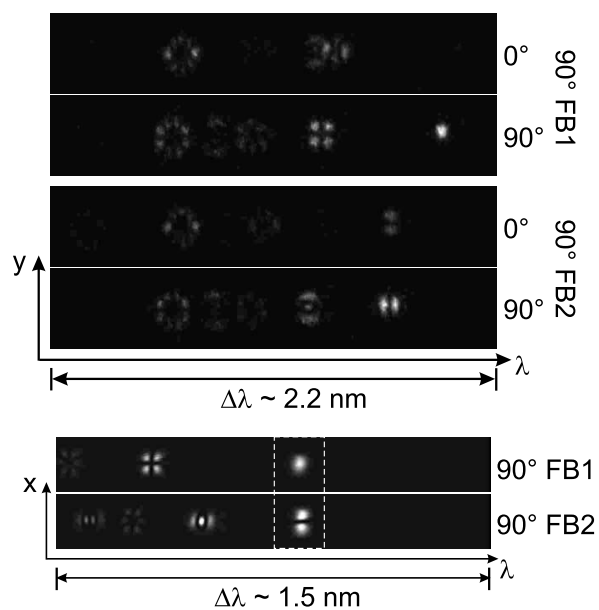

FIG. 5. Experimental single-shot measurements of polarizationresolved spectrally dispersed near-field profiles at $I_{\text {pump }}=4.5 \mathrm{~mA}$ with feedback of the fundamental mode $\mathrm{LP}_{01, y}(90 \circ \mathrm{FB} 1)$ and with feedback of the $\mathrm{LP}_{11, y}^{c}$ mode (90。 FB2). The mode selection by CCD2 is depicted in the lower part of the figure (note that the ordinate is $x$ in this subplot).

In the first pair of single-shot measurements $\left(90^{\circ} \mathrm{FB} 1\right)$, the selected fundamental mode is significantly enhanced. In addition, the optical spectra reveal that modes that do not contribute to the VCSEL's solitary emission (cf. Fig. 3) may be excited when feedback is applied. These modes emit especially if their spatial profile is complementary to the selected and enhanced mode's profile. For example, when the fundamental mode is fed back in $90^{\circ} \mathrm{FB} 1$, the $\mathrm{LP}_{41}$ modes in both polarizations are significantly enhanced, as they profit from the increase in the fundamental mode's intensity in the center of the VCSEL. Furthermore, the spectra reveal suppression of the fundamental mode in the $0^{\circ}$ polarization.

On the other hand, $90^{\circ} \mathrm{FB} 2$ depicts the spectral emission behavior when the $\mathrm{LP}_{11}^{c}$ mode in the $90^{\circ}$ polarization is fed back: the $\mathrm{LP}_{11}^{c}$ mode is enhanced in the $90^{\circ}$ polarization. In addition, the spatially complementary $\mathrm{LP}_{11}^{s}$ mode is enhanced, remarkably, in the perpendicular $0^{\circ}$ polarization. The $\mathrm{LP}_{11}$ modes coexist with each other, however, each of the two spatially complementary modes emits only in one of the two polarizations. In addition, due to the enhancement of the $\mathrm{LP}_{11}^{c}$ and $\mathrm{LP}_{11}^{s}$ modes, the competing $\mathrm{LP}_{21}$ modes in both polarizations are suppressed. The measurements therefore reveal a competition between the two polarizations under frequency-filtered feedback. Enhancing a certain mode by the feedback leads to enhancement of the spatially complementary mode preferably in the other polarization and not in the same polarization as the fed back mode.

Figure 6 depicts single-shot measurements, which demonstrate the effect of the two presented feedback conditions on the polarization-resolved near-field profiles. The effect of feeding back the fundamental mode in the $90^{\circ}$ polarization $\left(90^{\circ} \mathrm{FB} 1\right)$ on the near-field profile can be seen in the first row of Fig. 6. In the $90^{\circ}$ polarization, the contribution of the fed back Gaussian fundamental mode leads to an increase of the intensity in the central region compared to the solitary case (cf. Fig. 2). Correspondingly, in the $0^{\circ}$ polarization, the contribution of higher-order modes, such as the $\mathrm{LP}_{41}$ mode 


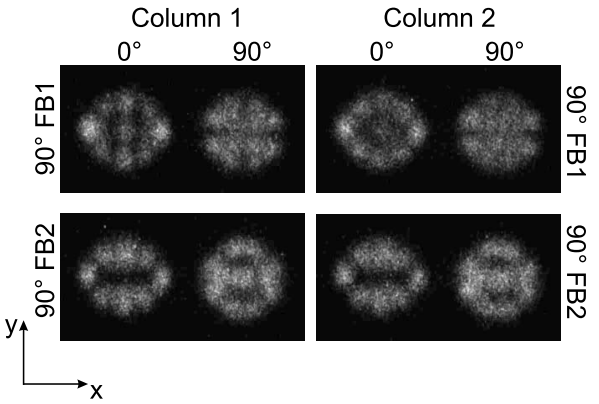

FIG. 6. Experimental single-shot measurements of the polarization-resolved near-fields at $I_{\text {pump }}=4.5 \mathrm{~mA}$, with feedback of the fundamental mode $\mathrm{LP}_{01, y}(90 \circ \mathrm{FB} 1)$ and with feedback of the $\mathrm{LP}_{11, y}^{c}$ mode (90。 FB2).

(cf. Fig. 5, $90^{\circ} \mathrm{FB} 1$ ), leads to pronounced peripheral (daisy mode) emission. A drastic modification of the near-field intensity distribution can also be observed when the $\mathrm{LP}_{11}^{c}$ mode $\left(90^{\circ} \mathrm{FB} 2\right)$ is fed back. The complementary emission can be observed well here, especially in the center of the aperture. The fed back $\mathrm{LP}_{11}^{c}$ mode in the $90^{\circ}$ polarization is superimposed by higher-order transverse modes, which also contribute to the $90^{\circ}$ polarization. Similarly, the emission in the $0^{\circ}$ polarization results from a superposition of the $\mathrm{LP}_{11}^{s}$ mode and higher-order modes, as illustrated in the corresponding optical spectra (Fig. 5). For both feedback conditions, a comparison of the two depicted single-shot measurements of the near-field profiles (columns 1 and 2) taken at different times does not reveal any significant differences in the intensity distributions. In contrast, the single-shot measurements of the solitary laser's near-field profiles showed significant variations in the intensity distributions (cf. Fig. 2). The similarity of the subsequently acquired single-shot measurements of the near-field profiles when a transverse mode is fed back suggests that the emission dynamics is considerably suppressed.

Three power spectra revealing the VCSEL's intensity dynamics, and especially, its polarization dynamics, are depicted in Fig. 7. For these, we used the linear polarizer LP2 (cf. Fig. 1) to select the desired polarization orientation. In Figs. 7(a)-7(c), the detected polarization orientations (selected by LP2) are $0^{\circ}, 90^{\circ}$, and $45^{\circ}$, respectively. The gray curves represent the emission without feedback (no FB), while the black curves represent the emission with feedback of the fundamental mode in the $90^{\circ}$ polarization orientation $\left(90^{\circ} \mathrm{FB} 1\right)$. Therefore, the power spectra in Fig. 7 compare the intensity dynamics with and without feedback. Figure 7 (a) reveals that the intensity dynamics in the $0^{\circ}$ polarization orientation are suppressed by up to $5 \mathrm{~dB}$ by the applied feedback. Similarly, Fig. 7(b) reveals suppression of the dynamics in the $90^{\circ}$ polarization orientation by up to $8 \mathrm{~dB}$. Finally, the effect of feeding back the fundamental mode in the $90^{\circ}$ polarization orientation on the combined emission dynamics of both polarization orientations (LP2: $45^{\circ}$ ) is depicted in Fig. 7(c). Here, considerable damping of the dynamical fluctuations in the overall emission is demonstrated. While the power spectra reveal that the intensity and polarization dynamics can indeed be reduced by feedback, the amount of suppression (in $\mathrm{dB}$ ) depends on the feedback conditions. For
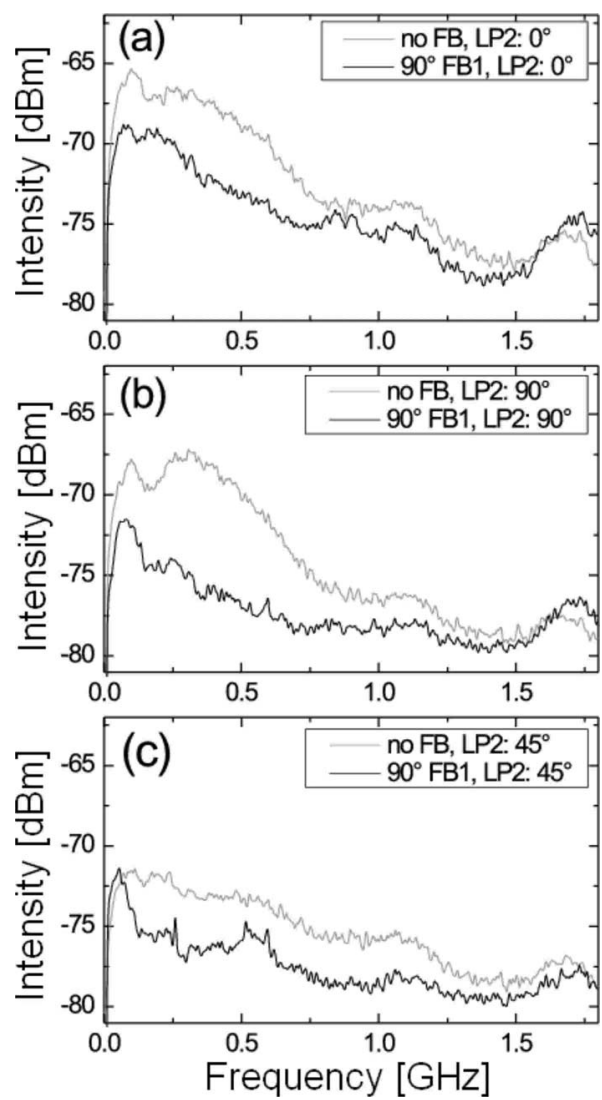

FIG. 7. Polarization-resolved rf-power spectra of the intensity dynamics at $I_{\text {pump }}=4.5 \mathrm{~mA}$. Gray: Without feedback (no FB); Black: With feedback of the fundamental mode $\left(\mathrm{LP}_{01}\right)$ in the $90^{\circ}$ polarization $\left(90^{\circ} \mathrm{FB} 1\right)$. Detected polarization orientation selected by LP2: (a) $0^{\circ}$, (b) $90^{\circ}$, and (c) $45^{\circ}$.

example, feedback of the fundamental mode in the $0^{\circ}$ polarization orientation $\left(0^{\circ} \mathrm{FB} 1\right)$ typically results in a much smaller suppression of the dynamics than shown here for feedback in the $90^{\circ}$ orientation. A possible origin of this difference may be that the feedback strength in the $0^{\circ}$ polarization orientation is lower than in the $90^{\circ}$ orientation (cf. Sec. II). Therefore, in the case of $0^{\circ}$ feedback, the resulting effective gain of the individual modes may not be sufficient to stabilize the emission. Furthermore, feedback-induced instabilities cannot be observed in the power spectra presented here. Though, in some cases, we could observe regular pulsations with a repetition rate corresponding to the external round-trip frequency $(\sim 260 \mathrm{MHz})$ when feedback was applied, the power spectra in Fig. 7 demonstrate that these pulsations can be avoided and the VCSEL's emission dynamics can be suppressed.

\section{MODEL}

The aim of this section is the derivation of deterministic evolution equations, which can describe the dynamics of a VCSEL submitted to polarization- and frequency-selective feedback. Such equations are intended to provide a theoretical understanding of the effect of this feedback on the spectral and dynamical emission properties of the laser. Full spa- 
tiotemporal models have yet been proposed for the modelization of solitary VCSELs, but however, despite their relatively high accuracy, they may not be computationally cost effective in our case since we would simultaneously have to take into account carrier diffusion, field diffraction, and the external time-delayed feedback.

An interesting alternative is to implement a modalexpansion model for the laser electric field. The advantage of such a modal model is that on the one hand, the simulations can be performed in a reasonable time, and on the other, the frequency shift of each transverse mode can be determined $a$ priori, so that we can easily match the central frequency of the grating to the frequency of the transverse mode we want to feed back into the laser.

The complex amplitude of the transverse electric field in each circular polarization can be expanded as

$$
\mathcal{E}_{ \pm}\left(\mathbf{r}_{\perp}, t\right)=\sum_{m, n} \Phi_{m n}\left(\mathbf{r}_{\perp}\right) E_{ \pm m n}(t) e^{i \omega_{m n} t},
$$

where $\mathbf{r}_{\perp}=(r, \varphi)$ is the transverse coordinate, and $E_{ \pm m n}(t)$ is the complex circular component of the modal amplitude associated with the transverse mode $\Phi_{m n}\left(\mathbf{r}_{\perp}\right)$. We also assume that the frequency shift of each transverse mode relative to the central optical carrier frequency $\Omega$ is $\omega_{m n}$.

The following sections will be devoted to the determination of the modal profiles, including the effects of geometrical and material anisotropies. Then, using an explicit filter profile for the frequency-selective grating, we will derive the coupled evolution equations for the system under study.

\section{A. Eigenmodes of a circular VCSEL}

The orthonormal eigenfunctions $\Phi_{m n}\left(\mathbf{r}_{\perp}\right)$ are the coldcavity modes determined by solving the eigenvalue problem

$$
\left[\nabla_{\perp}^{2}+\left(\frac{\Omega}{c}\right)^{2} 2 n_{e} \Delta n\right] \Phi_{m n}=\left(\frac{W_{m n}}{r_{w}}\right)^{2} \Phi_{m n},
$$

where $r_{w}$ is the waveguide radius, $n_{e}$ is the background refractive index, and $\Delta n$ is the step index variation between the core and the cladding of the VCSEL. The normalized solution of Eq. (2) is the $\mathrm{LP}_{m n}$ modes, which explicitly read

$$
\Phi_{m n}\left(\mathbf{r}_{\perp}\right)=A_{m n} e^{i m \varphi}\left\{\begin{array}{cc}
J_{m}\left[\left(r / r_{w}\right) \sqrt{V^{2}-W_{m n}^{2}}\right. & \text { if } r \leqslant r_{w} \\
J_{m}\left[\sqrt{V^{2}-W_{m n}^{2}}\right] & \\
\frac{K_{m}\left[\left(r / r_{w}\right) W_{m n}\right]}{K_{m}\left[W_{m n}\right]} & \text { if } r>r_{w},
\end{array}\right.
$$

where $A_{m n}$ is a normalization factor such that the modal profiles fulfill the orthonormalization condition

$$
\iint_{\infty} \Phi_{m n}\left(\mathbf{r}_{\perp}\right) \Phi_{k l}^{*}\left(\mathbf{r}_{\perp}\right) d^{2} \mathbf{r}_{\perp}=\delta_{m k} \delta_{n l} .
$$

In Eq. (3), $J_{m}$ and $K_{m}$ are the $m$ th order Bessel functions of the first and second kind, respectively, and $V$ $=(\Omega / c) r_{w} \sqrt{2 n_{e} \Delta n}$ is the waveguide parameter. A mode $\mathrm{LP}_{m n}$ has $2 m$ zeros in the azimuthal direction, and $n-1$ zeros in the radial one, so that, for example, the fundamental mode is $\mathrm{LP}_{01}$. The eigenvalues $W_{m n}$ are obtained through the resolution of the continuity equation for the derivative of the modal profiles at $r=r_{w}$, and amongst all the possible modes $\Phi_{m n}$, only the so-called guided modes satisfying $0<W_{m n}$ $<V$ can lase, with a frequency that is redshifted by an amount

$$
\omega_{m n}=-\frac{c^{2}}{2 \Omega n_{e} n_{g}}\left(\frac{W_{m n}}{r_{w}}\right)^{2},
$$

where $n_{g}$ is the group-velocity index.

In fact, the VCSEL aperture is never perfectly circular. This noncircularity (ellipticity in the simplest case) modifies the spatial extension of the transverse modes, and it can play a crucial role in the modal dynamics. The following section addresses this issue.

\section{B. Effect of ellipticity as a geometrical anisotropy}

A circular symmetry supports degenerated polarization modes with arbitrary spatial orientation. Ellipticity lifts this degeneracy by introducing two privileged spatial orientations for the modes, corresponding to the principal axes of the ellipse [13]. Hence, the degenerated mode $\Phi_{m n}\left(\mathbf{r}_{\perp}\right)$ $=\left|\Phi_{m n}\left(r / r_{w}\right)\right| e^{i m \varphi}$ with arbitrary spatial orientation will therefore be split into two spatially complementary modes, with fixed spatial orientation, respectively called the cosine mode and the sine mode, where the cos and sin functions will rule the azimuthal variations of the mode profiles.

Beyond pinning the modal orientation in the transverse plane, ellipticity also modifies the spatial shape of the modal profiles. For slightly elliptical cross sections, we can deduce the new modal profiles from those obtained for the circular cross-section resonator. Effectively, if we consider an elliptical aperture with half-axes $r_{a}>r_{b}$, we can in first approximation replace the reduced radius $r / r_{w}=\sqrt{\left(x / r_{w}\right)^{2}+\left(y / r_{w}\right)^{2}}$ in Eq. (3) by the "elliptical" reduced radius $\varrho$ $=\sqrt{\left(x / r_{a}\right)^{2}+\left(y / r_{b}\right)^{2}}$, so that the cosine and sine eigenmodes of the elliptical resonator can be expressed as the following real functions:

$$
\begin{aligned}
& \Phi_{m n}^{c}\left(\mathbf{r}_{\perp}\right)=\left[\delta_{m 0}+\sqrt{2}\left(1-\delta_{m 0}\right)\right]\left|\Phi_{m n}(\varrho)\right| \cos (m \varphi), \\
& \Phi_{m n}^{s}\left(\mathbf{r}_{\perp}\right)=\left[\delta_{m 0}+\sqrt{2}\left(1-\delta_{m 0}\right)\right]\left|\Phi_{m n}(\varrho)\right| \sin (m \varphi),
\end{aligned}
$$

where $\left[\delta_{m 0}+\sqrt{2}\left(1-\delta_{m 0}\right)\right]$ is a normalization factor equal to 1 when $m=0$, and to $\sqrt{2}$ when $m \neq 0$. It may be important to note that the modes $\mathrm{LP}_{m n}^{c}$ and $\mathrm{LP}_{m n}^{s}$ are spatially complementary.

The last effect of this geometrical anisotropy is the lifting of the frequency degeneracy of the spatial orientation modes. Therefore, a given mode may have two distinct eigenfrequencies for the two spatial orientations. The frequency splitting between spatial orientation modes generally amounts to a few tens of $\mathrm{GHz}[13,14]$, and we will here consider that these geometrically induced frequency shifts are equal to $\omega_{m n}^{c}=\omega_{m n}-\delta \omega$ and $\omega_{m n}^{s}=\omega_{m n}+\delta \omega$, respectively, with $\delta \omega / 2 \pi=5 \mathrm{GHz}$. 


\section{Material anisotropies}

The second type of anisotropies to consider are material anisotropies, namely, dichroism $\left(\gamma_{a}\right)$ and birefringence $\left(\gamma_{b}\right)$. Both have an electro-optical contribution induced by the voltage across the active medium, and an elasto-optical contribution induced by stress and strain exerted upon the crystalline structure of the semiconductor [14]. This latter contribution may be linked to ellipticity, as an elliptical aperture can also be considered as a circular domain with a positive stress in the direction of the short half-axis, and a symmetrical negative stress in the direction of the long half-axis. In the following, we assume that these mechanical constraints of opposite signs induce opposite sign shifts for the elastooptical contributions, i.e., different values for dichroism and birefringence according to the spatial orientation of the lasing mode. Therefore, the cosine modes may have anisotropies of the kind $\gamma_{a, b}^{c}=\gamma_{a, b}+\Delta \gamma_{a, b}$, while the sine modes will be subjected to anisotropies of the kind $\gamma_{a, b}^{s}=\gamma_{a, b}-\Delta \gamma_{a, b}$.

\section{Frequency-selective feedback}

A convenient way to take into account the frequency selection of the feedback has been to consider the grating as a frequency-selective mirror with a Lorentzian characteristic $[15,16]$, whose transfer function can be written as

$$
\mathcal{L}(\omega)=\frac{\Delta \omega_{G}}{\Delta \omega_{G}+i\left(\omega-\Omega_{G}\right)},
$$

where $\Omega_{G}$ is the central frequency of the grating, to be matched with the frequency $\Omega+\omega_{m n}$ of the fed back mode, and $\Delta \omega_{G}$ is the half-width at half-maximum quantifying the bandwidth of the filter. We will consider the case of narrow filters for which the bandwidth is smaller than the spacing between the lasing transverse modes $\left(\Delta \omega_{G} / 2 \pi=10 \mathrm{GHz}\right)$.

\section{E. Dynamical evolution equations}

The mesoscopic evolution equations under consideration are an extension of the modal-expansion model for VCSELs proposed in Ref. [5], which corresponds to a modal decomposition of the spatiotemporal model presented in Ref. [4]. The variables of the mesoscopic model are therefore the guided modes $E_{ \pm m n}^{c, s}(t)$, the spin-up and spin-down carrier densities $N_{ \pm}\left(\mathbf{r}_{\perp}, \bar{t}\right)$, and the filtered feedback variable $F_{m n}^{c, s}(t)$. A linear polarizer had been introduced in the external cavity; therefore, the rate equations, which are written in terms of circularly polarized modes, should take into account the fact that the fed back radiation is proportional to $E_{x, m n}^{c, s}=\left(E_{+m n}^{c, s}\right.$ $\left.+E_{-m n}^{c, s}\right) / \sqrt{2}$ if the polarizer is parallel to the $x$ direction (that is, the $0^{\circ}$ direction), and proportional to $E_{y, m n}^{c, s}=\left(E_{+m n}^{c, s}\right.$ $\left.-E_{-m n}^{c, s}\right) / i \sqrt{2}$ if the polarizer is parallel to the $y$ direction $\left(90^{\circ}\right.$ direction).

With these assumptions, we obtain the following equations for an $x$-polarized fed back mode:

$$
\begin{aligned}
\partial_{t} E_{ \pm \mu}^{c, s}= & \frac{1}{2}\left[-\kappa+(1+i \alpha) G_{ \pm \mu}^{c, s}\right] E_{ \pm \mu}^{c, s} \\
& -\left(\gamma_{a}^{c, s}+i \gamma_{b}^{c, s}\right) E_{\mp \mu}^{c, s}+\frac{K}{\sqrt{2}} F_{\mu}^{c, s},
\end{aligned}
$$

$$
\begin{gathered}
\partial_{t} F_{\mu}^{c, s}=-\left[\Delta \omega_{G}-i \varpi_{\mu}^{c, s}-i \Delta \Omega_{x}\right] F_{\mu}^{c, s} \\
+\frac{\Delta \omega_{G}}{\sqrt{2}}\left[E_{+\mu}^{c, s}(t-T)+E_{-\mu}^{c, s}(t-T)\right] e^{-i \theta_{\mu}^{c, s}}, \\
\partial_{t} N_{ \pm}=\frac{\eta_{i} I}{2 e W} C(\varrho)-\gamma_{e} N_{ \pm} \mp \gamma_{j}\left[N_{+}-N_{-}\right]+\mathcal{D} \nabla_{\perp}^{2} N_{ \pm} \\
-G_{ \pm}\left(N_{ \pm}\right) \sum_{\mu} \sum_{c, s}\left|\Phi_{\mu}^{c, s}\right|^{2}\left|E_{ \pm \mu}^{c, s}\right|^{2},
\end{gathered}
$$

where $\mu \equiv\{m, n\}$. The cavity losses are given by $\kappa$ $\left(=600 \mathrm{~ns}^{-1}\right)$, the linewidth enhancement factor $\alpha(=3.0)$, the spin-flip rate $\gamma_{j}\left(=100 \mathrm{~ns}^{-1}\right)$, the nonradiative recombination rate $\gamma_{e} \quad\left(=1.0 \mathrm{~ns}^{-1}\right)$, the bimolecular diffusion $\mathcal{D}$ $\left(=0.5 \mu \mathrm{m}^{2} \mathrm{~ns}^{-1}\right)$, the background refractive index $n_{e}(=3.3)$, the group-velocity refractive index $n_{g}(=3.5)$, the step-index variation between the core and the cladding $\Delta n(=0.01)$, and the optical carrier frequency $\Omega$ (deduced from $\lambda=850 \mathrm{~nm}$ ). The electro-optic contributions of dichroism and birefringence are $\gamma_{a}\left(=0.1 \mathrm{~ns}^{-1}\right)$ and $\gamma_{b}\left(=10 \mathrm{~ns}^{-1}\right)$, while the elastooptic shifts are $\Delta \gamma_{a}\left(=0.3 \mathrm{~ns}^{-1}\right)$ and $\Delta \gamma_{b}\left(=15 \mathrm{~ns}^{-1}\right)$.

The pumping current $I$ has been kept constant at $4.5 \mathrm{~mA}$; $\eta_{i}$ is the internal quantum efficiency $(=0.7)$ and $W$ is the thickness of the active region $(=32 \mathrm{~nm})$. We also assume that the injected electrons are uniformly distributed in the aperture of the active region, so that the normalized surface current density profile reads $C(\varrho)=1 / \pi r_{a} r_{b}$ if $\varrho<1$ and 0 otherwise.

The strength of the feedback is given by $K=[(1$ $\left.\left.-R_{\text {ext }}^{2}\right) / R_{\text {ext }}\right]\left(\rho / \tau_{\text {in }}\right)$, where $\tau_{\text {in }}$ is the round-trip time in the laser cavity $(=0.05 \mathrm{ps}), \rho$ is the external reflection amplitude (=3\%), and $R_{\text {ext }}$ is the external facet reflectivity of the VCSEL (=0.995). The external-cavity round-trip time is $T$ (=4 ns). In accordance with the dimensions of the VCSEL used in the experiments, the half-axis of the elliptical aperture are taken as $r_{a}=4.9 \mu \mathrm{m}$ and $r_{b}=4.4 \mu \mathrm{m}$. With the above parameters, the eigenspectrum $\omega_{m n}$ corresponding to the optical cavity can be calculated with the mean radius $r_{m}=\left(r_{a}\right.$ $\left.+r_{b}\right) / 2$, so that the modal optical phase is $\theta_{m n}=\left(\Omega+\omega_{m n}\right) T$, and the modal frequency detuning relatively to the fed back mode $\omega_{\mu_{0}}^{c, s}$ is $\varpi_{\mu}=\omega_{\mu_{0}}^{c, s}-\omega_{\mu}^{c, s}$.

The material gain is defined as $G_{ \pm}\left(N_{ \pm}\right)=g\left[2 N_{ \pm}-N_{0}\right]$, where $g\left(=2.6 \times 10^{-13} \mathrm{~m}^{3} \mathrm{~s}^{-1}\right)$ is the differential gain, and $N_{0}$ $\left(=1.5 \times 10^{24} \mathrm{~m}^{-3}\right)$ is the carrier density at transparency. The modal gain $G_{\mu}$ is therefore defined as

$$
G_{ \pm \mu}^{c, s}=\iint_{\mathcal{S}} G_{ \pm}\left(N_{ \pm}\right)\left|\Phi_{\mu}^{c, s}\left(\mathbf{r}_{\perp}\right)\right|^{2} d^{2} \mathbf{r}_{\perp} .
$$

At last dichroism, birefringence, and linewidth enhancement factor induce a global frequency blueshift equal to $\Delta \Omega_{x}$ $=\alpha \kappa / 2-\left(\gamma_{b}-\alpha \gamma_{a}\right)$ in the case of $x$-polarized feedback, and to $\Delta \Omega_{y}=\alpha \kappa / 2+\left(\gamma_{b}-\alpha \gamma_{a}\right)$ in the case of $y$-polarized feedback. In the case of $y$-polarized feedback, the feedback coefficient should be written as $\pm i K_{m n} / \sqrt{2}$, instead of $K_{m n} / \sqrt{2}$ as for $x$-polarized feedback.

This model enables us to simulate the instantaneous complex amplitudes of the various modes $\mathrm{LP}_{m n}$, which are characterized by their polarization ( $x$ or $y$ ), and by their spatial 
orientation $(c$ or $s)$. In the numerical simulations, all these modes are simultaneously taken into account.

\section{MODEL RESULTS}

\section{A. Analysis of the influence of polarization- and frequency-selective feedback}

We here evaluate the effect of feedback on the modal gain of the various transverse modes. We set in first approximation $\left|E_{+}\right|=\left|E_{-}\right| \equiv|E| / \sqrt{2}, \quad N_{+}=N_{-} \equiv N / 2$, and introduce a unique material gain function $G(N)=g\left(N-N_{0}\right)$. The final stationary states can then be expressed as

$$
\begin{gathered}
E_{\mu}(t)=\sqrt{P_{\mu}} e^{i \Delta \Omega_{x} t} e^{i \Delta \omega t}, \\
F_{\mu}(t)=\sqrt{Q_{\mu}} e^{i \Delta \Omega_{x} t} e^{i \Delta \omega t} e^{i \sigma_{\mu}},
\end{gathered}
$$

where $\Delta \omega$ stands for the external-cavity modes (ECMs). Inserting these expressions in Eqs. (8) and (9), we can, after some algebra, derive the transcendental algebraic equation for these ECMs according to

$$
\Delta \omega=\frac{K \sqrt{1+\alpha^{2}}}{\sqrt{1+\left(\frac{\varpi_{\mu}-\Delta \omega}{\Delta \omega_{G}}\right)^{2}}} \sin \left[\sigma_{\mu}-\arctan \alpha\right],
$$

with

$$
\sigma_{\mu}=-\left(\Delta \Omega_{x}+\Delta \omega\right) T-\theta_{\mu}+\arctan \left(\frac{\varpi_{\mu}-\Delta \omega}{\Delta \omega_{G}}\right) .
$$

By resolving the above equation, it is possible to find the frequencies $\Delta \omega$ of the ECMs associated with each transverse mode $\mu$.

A given mode does lase if and only if $\sqrt{P_{\mu}}$ and $\sqrt{Q_{\mu}}$ are different from 0 . If we consider the real parts in Eqs. (8) and (9), we obtain

$$
\begin{gathered}
\frac{1}{2}\left[-\kappa+G_{\mu}-\gamma_{a}\right] \sqrt{P_{\mu}}+K \sqrt{Q_{\mu}} \cos \sigma_{\mu}=0, \\
\sqrt{P_{\mu}} \cos \left[\sigma_{\mu}+\left(\Delta \Omega_{x}+\Delta \omega\right) T+\theta_{\mu}\right]-\sqrt{Q_{\mu}}=0,
\end{gathered}
$$

and the lasing condition (nonzero $\sqrt{P_{\mu}}$ and $\sqrt{Q_{\mu}}$ ) imposes to equate to zero the corresponding determinant, thereby leading to

$$
G_{\mu}=\kappa+\gamma_{a}-\frac{2 K \cos \sigma_{\mu}}{\sqrt{1+\left(\frac{\varpi_{\mu}-\Delta \omega}{\Delta \omega_{G}}\right)^{2}}} .
$$

The above equation is a key result: It shows that due to the feedback, the modal gain is either increased or decreased for each transverse mode. However, this effect of the feedback is noticeable only for the mode selected by the grating (the one for which $\varpi \simeq 0$ ); in that case, the gain can be increased or decreased by an amount of the order of $1-5 \%$. The modal gains of the other modes are not directly affected by the frequency-selective feedback (FSF), because their detuning $\varpi$ relative to the central frequency of the filter is too large

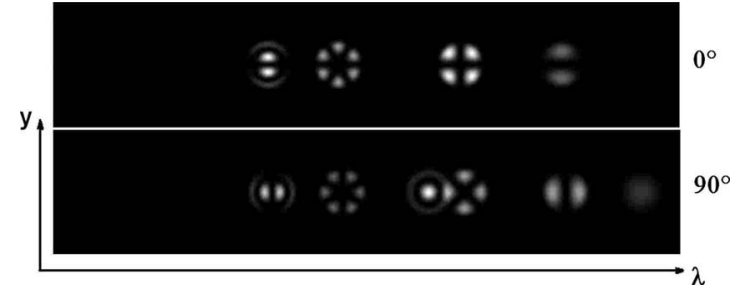

FIG. 8. A numerical snapshot picture of the spectrally resolved near-field intensity distributions of the VCSEL without FSF, for $I=4.5 \mathrm{~mA}$.

relative to $\Delta \omega_{G}$. It is also interesting to note that according to Eq. (18), a suitable parameter, which can enable to increase or decrease the gain, is the feedback phase $\theta_{\mu}=\left(\Omega+\omega_{\mu}\right) T$. In particular, tuning the laser central frequency $\Omega$ within the span of the external-cavity spacing frequency $\omega_{\text {ext }}=2 \pi / T$ can lead to an increase or a decrease of the modal gains through the sign of $\cos \sigma_{\mu}$.

The above result can also explain how a small amount of feedback can lead to a drastic redistribution of modal output powers. In Ref. [17], Valle and Pesquera have shown that in a VCSEL without feedback, the stationary optical output powers $P_{\mu}$ of the guided transverse modes obey a linear set of algebraic equations

$$
\sum_{\nu} a_{\mu \nu} P_{\nu}=b_{\mu}
$$

where the coefficients $a_{\mu \nu}$ and $b_{\mu}$ are constants. However, the solutions $P_{\mu}$ of this set of equations should absolutely be positive. Therefore, if by solving Eq. (19) a negative value is found for a given mode $P_{\nu}$, this mode must be discarded (following a precise procedure) and a new linear set of equations of lower dimensionality corresponding to the remaining modes must then be solved, until only strictly positive solutions are obtained for all the lasing modes.

From the theory presented above, we can understand why the FSF can affect several modes even though it directly affects only the fed back mode $\mu_{0}$ for which $\varpi_{\mu_{0}} \simeq 0$. In fact, in Eq. (19) the difference between a VCSEL without FSF and another with FSF is a perturbation (proportional to $K$ ) of the parameters $a_{\mu \mu_{0}}$ and $b_{\mu_{0}}$. Before the perturbation is applied, we have exclusively positive solutions (lasing modes), but when this feedback perturbation is applied, some of these solutions turn to be negative. This is the mechanism that explains how a small perturbation only affecting one mode can lead to the suppression, excitation, or amplitude variation of various others modes.

\section{B. Numerical simulations}

The essential features of the experimentally observed behavior were recovered during the numerical simulations.

In Fig. 8, the numerical near-field spectrum of the VCSEL is represented and it presents a strong similarity with the experimental spectrum of Fig. 3. The spectral splitting of these transverse modes, which is determined through Eq. (5), is in excellent agreement with the experimental measurements. It can be theoretically demonstrated that the modes of 


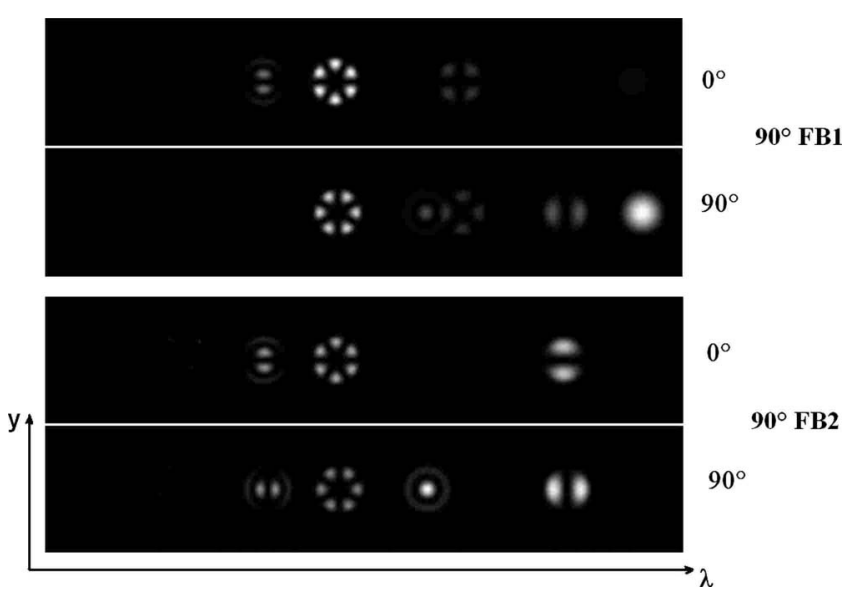

FIG. 9. A numerical snapshot picture of polarization-resolved spectrally dispersed near-field profiles at $I_{\text {pump }}=4.5 \mathrm{~mA}$ with feedback of the fundamental mode $\mathrm{LP}_{01, y}\left(90^{\circ} \mathrm{FB} 1\right)$ and with feedback of the $\mathrm{LP}_{11, y}^{c}$ mode $\left(90^{\circ} \mathrm{FB} 2\right)$.

same order $\mathcal{O}=m+2 n-1$ are nearly frequency degenerated: Hence, the modes $\mathrm{LP}_{01}$ (fundamental mode) and $\mathrm{LP}_{11}$ (second-order mode) are spectrally isolated, while for example, the third-order modes $\mathrm{LP}_{21}$ and $\mathrm{LP}_{02}$ are almost superimposed in the experimental and numerical spectra.

One can note in Fig. 8 that the fundamental mode lases very weakly (in only one polarization), while several higherorder modes are strongly excited, in both polarizations. It can also be seen that free lasing modes (without feedback) are, in general, spatially complementary in order to deplete all the available carriers, and also complementary in polarizations. This double complementarity can be observed both experimentally and numerically. Ellipticity also plays a role, as it favors the modes that are aligned along the longest half-axis, because these modes profit from a larger spatial overlap over the active medium. This can be clearly seen in the experimental spectra for the "two-lobe" modes $\mathrm{LP}_{11}$ without feedback. The modeling of ellipticity successfully enables one to recover this modal discrimination, as the numerical simulations clearly replicate this experimental feature.

The effect of frequency-selective feedback can be observed in Fig. 9. Here again, the numerical spectra are quite similar to the experimental spectra of Fig. 5 in both polarizations. The only noticeable difference is that the $\mathrm{LP}_{41}$ is excited experimentally while it is the $\mathrm{LP}_{31}$ mode that is excited numerically. However, these two modes are both highorder transverse modes with approximately the same qualitative features, so the apparent difference is not very important. Typically, it can be observed that a fed back mode can always be amplified if it has the suitable feedback phase. Note, for example, that at this value of $I_{\text {pump }}$, the fundamental mode is almost switched off without feedback, but it lases strongly with feedback. Experiments, numerics, and analytics coincide on that point. In addition, a mode lasing strongly without feedback can be practically switched off when the feedback is applied, as is the case, for example, for the modes $\operatorname{LP}_{21}(c$ and $s$ ). Finally, it should also be noted that when a given mode is amplified, the spatially complementary mode is also amplified, with the opposite polarization, and here again, there is a clear agreement between theory and experience.

\section{CONCLUSION AND OUTLOOK}

The influence of polarization- and frequency-filtered optical feedback on the emission behavior of a multimode $10 \mu \mathrm{m}$ VCSEL has been studied experimentally and theoretically. The applied feedback configuration allows selection and feedback of single transverse modes, by which the modal composition of the optical spectrum is affected.

It has been shown analytically that the modal gains are tightly related to the distribution of external-cavity modes, which are themselves dependent on the spectral properties of the external-cavity filter. It has therefore been possible to demonstrate that the optical phase attached to each transverse mode plays a decisive role as far as the enhancement or the quenching of a mode is concerned. Numerical simulations have shown good agreement with experimental measurements, in terms of spatial modal profiles and dynamics, and in terms of specific emission properties such as polarization and spatial complementarity. By selectively enhancing an individual mode, spatially complementary modes are also enhanced preferably in the polarization perpendicular to the fed back mode. Finally, our experimental and theoretical investigations have shown that of the emission dynamics of the VCSEL can be substantially stabilized when this type of feedback is applied.

There are interesting perspectives of future investigations on this topic, particularly on the nonlinear dynamics aspect. It is known that delayed feedback has an ambivalent nature, as it may stabilize or destabilize a dynamical system; here, the stabilization feature is prevalent, but the conditions under which a "coherence collapse"-like behavior can emerge are still to be determined. Another fundamental aspect is the study of the interaction between delayed feedback and spatial extension. By addressing individual modes that have a given spatial overlap, frequency-selective feedback applied on broad-area VCSELs can be a very useful tool in order to investigate and understand this interaction.

\section{ACKNOWLEDGMENTS}

S.K.M., I.F., and W.E. thank the Volkswagen Foundation for financial support. Y.K.C. acknowledges financial support through a research grant from the Govern de les Illes Balears. Furthermore, the authors thank Infineon Technologies AG for providing the excellent VCSEL structures. They also acknowledge fruitful discussions with Dr. J. Mulet. 
[1] H. Soda, K.-I. Iga, C. Kitahara, and Y. Suematsu, Jpn. J. Appl. Phys. 18, 2329 (1979).

[2] A. Barchanski, T. Gensty, C. Degen, I. Fischer, and W. Elsässer, IEEE J. Quantum Electron. 39, 850 (2003).

[3] C. J. Chang-Hasnain, J. P. Harbison, G. Hasnain, A. C. Von Lehmen, L. T. Florez, and N. G. Stoffel, IEEE J. Quantum Electron. 27, 1402 (1991).

[4] J. Mulet and S. Balle, IEEE J. Quantum Electron. 38, 291 (2002).

[5] J. Mulet and S. Balle, Phys. Rev. A 66, 053802 (2002).

[6] C. Degen, W. Elsässer, and I. Fischer, Opt. Express 5, 38 (1999).

[7] K. Becker, I. Fischer, and W. Elsässer, Proc. SPIE 5452, 452 (2004).

[8] D.-L. Cheng, E.-C. Liu, and T.-C. Yen, IEEE Photonics Technol. Lett. 16, 278 (2002).

[9] F. Marino, S. Barland, and S. Balle, IEEE Photonics Technol.
Lett. 15, 789 (2003).

[10] Y. Tanguy, N. Radwell, T. Ackemann, and R. Jager, Phys. Rev. A 78, 023810 (2008).

[11] M. G. Littman, Opt. Lett. 3, 138 (1978).

[12] C. Degen, B. Krauskopf, G. Jennemann, I. Fischer, and W. Elsässer, J. Opt. B: Quantum Semiclassical Opt. 2, 517 (2000).

[13] K. D. Choquette and R. E. Leibenguth, IEEE Photonics Technol. Lett. 6, 40 (1994).

[14] P. Debernardi, G. P. Bava, C. Degen, I. Fischer, and W. Elsässer, IEEE J. Quantum Electron. 38, 73 (2002).

[15] B. Tromborg, H. Olesen, X. Pan, and S. Saito, IEEE J. Quantum Electron. 23, 1875 (1987).

[16] M. Yousefi and D. Lenstra, IEEE J. Quantum Electron. 35, 970 (1999).

[17] A. Valle and L. Pesquera, J. Opt. Soc. Am. B 19, 1549 (2002). 